\title{
Recomendações de Melhoria Baseadas na Cultura Organizacional para Iniciativas em Melhoria de Processo de Software
}

\author{
Odette M. Passos ${ }^{1}$, Arilo C. Dias-Neto ${ }^{2}$, Raimundo da S. Barreto ${ }^{2}$ \\ ${ }^{1}$ Instituto de Ciências Exatas e Tecnologia - Universidade Federal do Amazonas \\ Rua Nossa Senhora do Rosário, 3863 - 69100-000 - Tiradentes \\ Itacoatiara - AM - Brasil \\ ${ }^{2}$ Instituto de Computação - Universidade Federal do Amazonas \\ Av. General Rodrigo Otávio, 3000 - 69077-000 - Coroado \\ Manaus - AM - Brasil \\ \{odette, arilo, rbarreto\} eicomp.ufam.edu.br
}

\begin{abstract}
In the context of software organizations, organizational culture has been observed in scientific research as one of the critical factors for successful implementation of Software Process Improvement (SPI) initiatives. Therefore, this work presents a conceptual framework that based on the analysis of organizational culture obtained from a software organization, suggests recommendations for improvement that can be implemented in the organization aiming at positively supporting the implementation of SPI initiatives. Its evaluation was performed in two stages, initially with software engineers from four Brazilian software organizations evaluated positively the applicability of the proposed framework to support the implementation of SPI initiatives, secondly with appraisers and consultants of SPI initiatives, that evaluated positively the organizational diagnostic provided by the framework.
\end{abstract}

Resumo. No contexto de organizações de software, a cultura organizacional tem sido observada como sendo um dos fatores críticos para o sucesso da implementação de iniciativas de Melhoria de Processo de Software (MPS). Diante disso, esse artigo apresenta um framework conceitual que, a partir da análise da cultura organizacional de uma organização de software, sugere recomendações de melhoria que podem ser implantadas com o intuito de apoiar positivamente as implementações de iniciativas de MPS. A sua avaliação foi feita em duas etapas, inicialmente com profissionais de quatro empresas de software brasileiras, que avaliaram positivamente a aplicabilidade do framework no apoio à implementação de iniciativas de MPS, e em seguida com avaliadores e consultores de iniciativas de MPS, que avaliaram positivamente o diagnóstico organizacional provido pelo framework.

\section{Introdução}

Nos últimos anos, muitas organizações de software têm investido seus recursos em iniciativas de melhoria de processo de software (MPS) de forma a garantir a sua sobrevivência em um mundo cada vez mais competitivo e com clientes altamente exigentes. Essas iniciativas proveem uma sequência de passos para que um processo de desenvolvimento apresente um alto nível de excelência na qualidade de seus produtos [Shih e Huang 2010; Dyba 2005].

Por outro lado, a cultura organizacional $(\mathrm{CO})$ é um fator que pode influenciar a forma como os profissionais reagem a iniciativas de MPS [Dyba 2002]. A organização precisa utilizar os insights da CO para melhor implementar essas iniciativas, isto é, a alta 
direção precisa assegurar que as práticas de MPS estejam de acordo com a CO estabelecida na organização, e assim impedir ou prevenir o fracasso da iniciativa de MPS adotada pela organização [Muller et al. 2009].

Diante desse contexto, o objetivo deste trabalho é apresentar o desenvolvimento de um framework conceitual que a partir da análise da CO de uma organização de software, sugira recomendações de melhoria (RMs) que podem ser implantadas com o intuito de apoiar positivamente as implementações de iniciativas de MPS.

Para avaliar o framework proposto, foi conduzida uma investigação quantitativa, inicialmente com profissionais de quatro empresas de software brasileiras, com a finalidade de avaliar o questionário usado no framework para capturar a $\mathrm{CO}$ da organização que deseja implantar a iniciativa de MPS. Uma segunda avaliação, desta vez qualitativa, foi realizada com avaliadores e consultores de iniciativas de MPS a fim de avaliar o diagnóstico organizacional que contêm o perfil (CO) da organização e as RMs adequadas que visam apoiar as iniciativas de MPS. Os resultados de ambos os estudos indicam que o framework é pertinente para auxiliar a organização no entendimento dos valores organizacionais e no auxílio a implementação de iniciativas de MPS.

O texto está dividido em seis seções, considerando esta Introdução. A Seção 2 apresenta os conceitos fundamentais necessários ao desenvolvimento do framework. A Seção 3 apresenta os estudos realizados e os resultados obtidos para a construção do framework. A Seção 4 apresenta a estrutura do framework, enquanto que a Seção 5 descreve a sua avaliação. Finalmente, a Seção 6 apresenta as considerações finais e as principais contribuições do trabalho.

\section{Principais Conceitos Relacionados}

A CO refere-se a um sistema de valores, compartilhado pelos membros de uma organização, e que a difere de uma para outra. Esse sistema é, em última análise, um conjunto de características chaves que a organização valoriza [Robbins 2005]. Os trabalhos publicados em [Shih e Huang, 2010; Muller et al. 2009, 2010] indicam a CO como um dos fatores principais que podem influenciar a forma de como os profissionais reagem a iniciativas de MPS. Além disso, a CO pode ainda ajudar a explicar o sucesso ou o fracasso das organizações na implantação de tais iniciativas, assim como sua implicação na motivação e desempenho dos seus colaboradores.

Um dos componentes importantes da CO são os valores organizacionais (VOs), que constituem o núcleo da $\mathrm{CO}$ e são elementos fundamentais na construção e no desenvolvimento da identidade de uma empresa. Os VOs são incorporados dentro das organizações dando uma característica de estabilidade que é semelhante aos valores individuais e sociais [Webster 2010]. Por isso, é muito importante identificar e avaliar os VOs, pois estes são componentes importantes da CO [Schein 2009] e orientam o funcionamento da organização e de seus membros [Seevers 2000].

Estudos experimentais foram conduzidos nos últimos anos visando identificar as motivações e dificuldades para o sucesso ou fracasso na adoção de uma iniciativa de MPS. Porém, em geral, esses estudos tiveram um foco mais generalizado, se reportando a essas motivações e dificuldades como "fatores críticos de sucesso" (FCS) em iniciativas de melhoria [Dyba 2000; Montoni e Rocha 2010; Nasir et al. 2008; Niazi et al. 2005]. Muitos desses FCS dizem respeito às questões organizacionais, o que reforça a premissa de que a $\mathrm{CO}$ seria um aspecto importante para se obter sucesso na implantação de iniciativas de MPS. 
Organizações costumam se basear em RMs disponíveis, geralmente, na literatura técnica, para apoiar a implementação de iniciativas de MPS. As RMs são sugestões que ajudam as organizações na tomada de decisões e normalmente são baseadas em experiências anteriores [Barreto e Rocha 2012]. Elas têm como objetivo fornecer possíveis orientações relacionadas a aspectos considerados relevantes para uma organização, potencializando os resultados esperados e, até mesmo, garantindo sua permanência no mercado. No entanto, estas RMs podem apresentar direcionamentos sob diferentes perspectivas, sejam organizacionais, técnicas, culturais, humanas, ferramentais, dentre outras possibilidades. Neste trabalho, o escopo está direcionado à análise da perspectiva da CO sobre as iniciativas de MPS.

\section{Estudos Realizados e Resultados Obtidos para a Construção do Framework}

O método de pesquisa adotado neste trabalho, para a construção do framework proposto, está fundamentado nos princípios da Engenharia de Software Experimental. Ele se baseia na condução de estudos primários (por meio de pesquisa de opinião survey) e secundários (por meio de revisão sistemática) [Spínola et al. 2008] e foi dividido em duas fases, conforme mostrado na Figura 1.

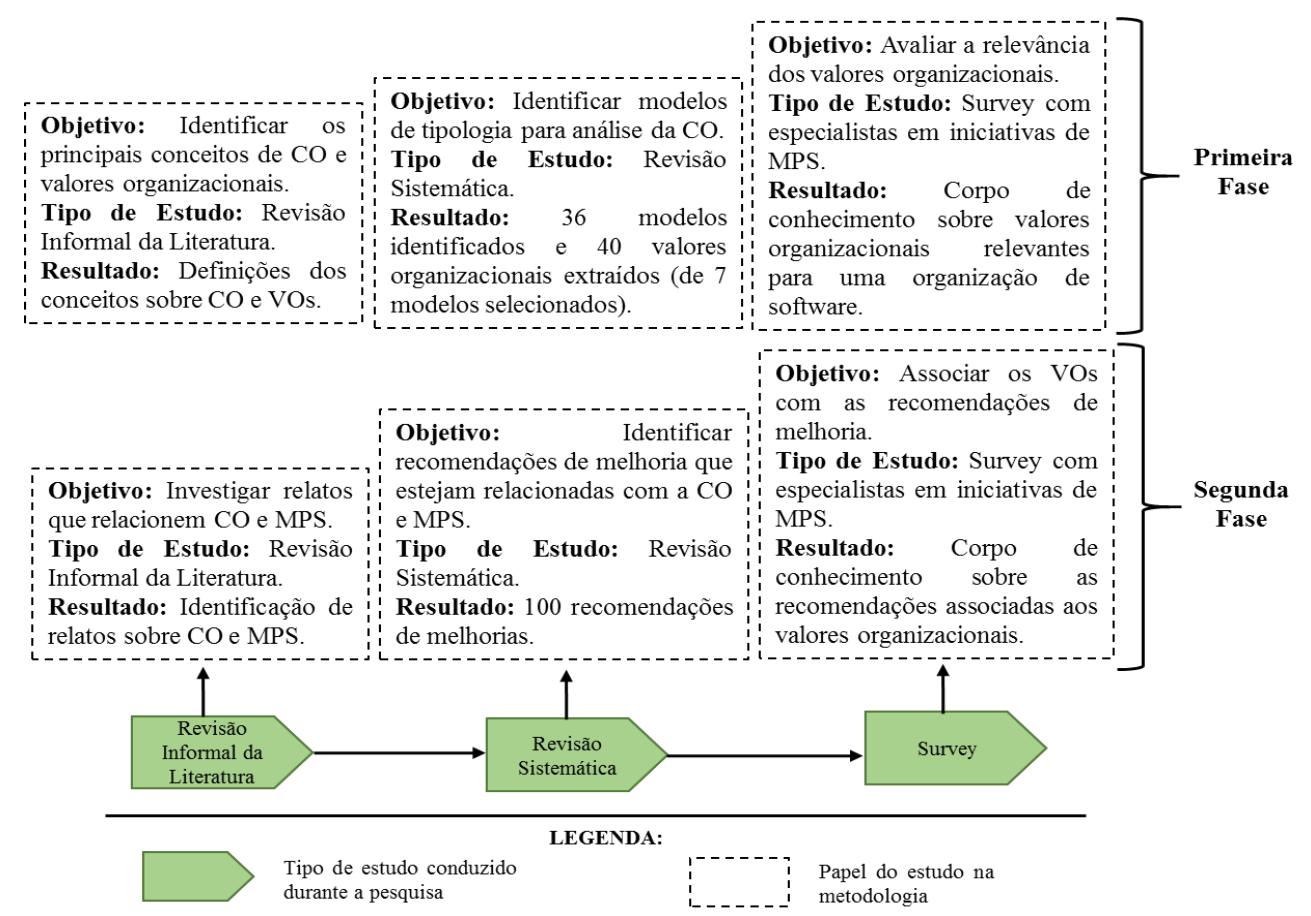

Figura 1. Resumo dos estudos conduzidos na primeira e segunda fase da pesquisa

\subsection{Primeira Fase da Pesquisa}

Nesta fase, uma revisão sistemática foi conduzida no período de janeiro a abril de $2011 \mathrm{e}$ teve como objetivos: Identificar e conhecer os modelos de tipologias existentes na literatura para análise da $\mathrm{CO}$ e Extrair os principais VOs abordados por cada modelo.

No total, foram identificados 36 modelos de tipologias para análise da $\mathrm{CO}$, dos quais 7 modelos foram selecionados. Após essa etapa, foi possível determinar um conjunto inicial de 40 VOs (veja Tabela 1) que podem caracterizar a CO de uma empresa. Eles foram extraídos a partir da definição das características tipológicas citadas por cada modelo selecionado e pela descrição do método quantitativo utilizado para se 
chegar a essa tipologia. Os VOs foram distribuídos em três categorias que representam o tipo de profissional da organização responsável pela sua implantação [Niazi et al. 2005]:

- Alta Gerência (AG): representada pela alta gerência e executivos, com 20 VOs $(50 \%)$

- Líder de Projetos (LP): representado por gerente de projeto e coordenador, com 7 VOs (17\%);

- Desenvolvedores (DE): representados pela equipe técnica do projeto (projetistas, testadores, programadores e analistas), com 13 VOs (33\%).

A distribuição dos VOs nas categorias foi baseada na leitura da definição das características tipológicas que sugerem a classe em que o VO está associado, como por exemplos, grupos de trabalho, líderes, gerentes e organização. Essas classes foram adaptadas para o tipo de profissional específico que trabalha na organização de software, como, alta gerência, líder de projetos e desenvolvedores.

A partir desse resultado, um survey foi planejado e executado visando identificar a importância de cada VO para caracterizar a CO em uma organização de software e o nível de relevância para uma organização de software que esteja envolvida em uma iniciativa de MPS. Participaram deste estudo 41 profissionais/pesquisadores. Os detalhes desta fase da pesquisa podem ser obtidos em [Passos et al. 2012]. A Tabela 1 apresenta os resultados obtidos nesta primeira fase.

\subsection{Segunda Fase da Pesquisa}

Nesta fase, uma segunda revisão sistemática foi conduzida no período de fevereiro a agosto de 2012 e teve como objetivos: Investigar o impacto da CO (se o resultado é positivo ou negativo) em iniciativas de MPS nas organizações de software e Extrair recomendações de melhoria que relacionam $\mathrm{CO}$ e iniciativas de MPS.

Com relação ao primeiro objetivo, das 28 publicações selecionadas para o estudo, que envolviam uma iniciativa de MPS e faziam referências a CO, 60,7\% (17 publicações) não citam se a relação entre CO e iniciativas de MPS foi positiva ou negativa, isto é, se a cultura influenciou ou não na adoção de uma iniciativa de MPS. Por outro lado, 28,6\% (8 publicações) citam que a $\mathrm{CO}$ influenciou positivamente na adoção da iniciativa e 10,7\% (3 publicações) citam que a influência foi negativa.

As 8 publicações cujo impacto foi positivo comentam que (i) as novas ideias ou técnicas não irão funcionar corretamente até que estejam alinhadas com a CO (2 publicações), (ii) o entendimento sobre as questões organizacionais é tão importante quanto à tecnologia para que se tenha sucesso em uma iniciativa de MPS (1 publicação) e que iii) a CO tem um papel fundamental no sucesso da implementação da iniciativa de MPS (5 publicações).

Com relação ao segundo objetivo, das 11 publicações identificadas, foi possível extrair 100 RMs (veja uma parte das RMs na Tabela 2), que estão relacionadas com a $\mathrm{CO}$ em uma iniciativa de MPS. As RMs foram organizadas em grupos que representam esses fatores (humano, social e organizacional) no processo de implementação da iniciativa de MPS. Para facilitar a organização das RMs identificadas, cada grupo (fator) foi dividido em categorias que foram determinadas conforme a palavra-chave retirada do texto da própria RM. Assim, o fator humano (FH) foi subdividido em 3 categorias, o fator social (FS) em 5 categorias e o fator organizacional (FO), em 5 categorias. Maiores detalhes podem ser obtidos em [Passos et al. 2013]. 
Tabela 1. Avaliação da Importância e Relevância dos VOs

\begin{tabular}{|c|c|c|c|c|}
\hline ID & Cat. & Valores Organizacionais & $\begin{array}{c}\text { Nível de } \\
\text { Importância }\end{array}$ & $\begin{array}{l}\text { Nível de } \\
\text { Relevância }\end{array}$ \\
\hline VO1 & $\mathrm{AG}$ & $\begin{array}{l}\text { Política de compromisso com a qualidade dos produtos, } \\
\text { serviços e processos }\end{array}$ & $100,0 \%$ & $91,2 \%$ \\
\hline VO2 & $\mathrm{AG}$ & Visão, metas e objetivos claros e estabelecidos & $100,0 \%$ & $90,2 \%$ \\
\hline VO3 & $\mathrm{DE}$ & Responsabilidades quanto a prazos e metas & $100,0 \%$ & $89,0 \%$ \\
\hline VO4 & LP & Informação das decisões tomadas & $100,0 \%$ & $75,5 \%$ \\
\hline VO5 & $\mathrm{AG}$ & Sistema de remuneração adequado ao cargo ocupado & $100,0 \%$ & $63,4 \%$ \\
\hline VO6 & LP & Acompanhamento das atividades planejadas & $98,4 \%$ & $88,5 \%$ \\
\hline VO7 & $\mathrm{AG}$ & Capacidade de se auto reorganizar para mudanças & $98,4 \%$ & $78,6 \%$ \\
\hline VO8 & $\mathrm{AG}$ & Investimento em tecnologia e inovação & $97,0 \%$ & $65,1 \%$ \\
\hline VO9 & $\mathrm{DE}$ & Envolvimento, comprometimento e participação & $96,6 \%$ & $87,6 \%$ \\
\hline VO10 & $\mathrm{DE}$ & Capacidade de trabalhar em grupo & $96,6 \%$ & $82,8 \%$ \\
\hline VO11 & $\mathrm{DE}$ & Capacidade de se adaptar a mudanças & $96,6 \%$ & $73,9 \%$ \\
\hline VO12 & $\mathrm{DE}$ & Cooperativismo e colaborativismo & $96,5 \%$ & $81,3 \%$ \\
\hline VO13 & $\mathrm{AG}$ & Plano de gerenciamento estratégico & $96,3 \%$ & $81,1 \%$ \\
\hline VO14 & $\mathrm{AG}$ & $\begin{array}{l}\text { Investimento em cursos de aperfeiçoamento, capacitação } \\
\text { e treinamento }\end{array}$ & $95,7 \%$ & $77,2 \%$ \\
\hline VO15 & $\mathrm{DE}$ & $\begin{array}{l}\text { Compartilhamento de valores, como respeito, lealdade e } \\
\text { ética }\end{array}$ & $95,7 \%$ & $75,8 \%$ \\
\hline VO16 & $\mathrm{AG}$ & Nível de relacionamento entre os funcionários & $94,9 \%$ & $65,6 \%$ \\
\hline VO17 & AG & Valorizar e encorajar idéias criativas e inovadoras & $93,7 \%$ & $64,6 \%$ \\
\hline VO18 & $\mathrm{DE}$ & Participação na definição das metas e objetivos & $92,2 \%$ & $66,8 \%$ \\
\hline VO19 & $\mathrm{DE}$ & Competência & $91,4 \%$ & $76,6 \%$ \\
\hline VO20 & $\mathrm{DE}$ & $\begin{array}{l}\text { Facilidades de aceitação em relação a pessoas externas e } \\
\text { novos funcionários }\end{array}$ & $91,4 \%$ & $57,7 \%$ \\
\hline VO21 & $\mathrm{AG}$ & Estrutura organizacional definida & $91,3 \%$ & $71,5 \%$ \\
\hline $\mathrm{VO} 22$ & LP & Disponibilidade e acessibilidade & $90,8 \%$ & $61,3 \%$ \\
\hline VO23 & $\mathrm{AG}$ & Estratégia para gerenciamento dos riscos & $90,0 \%$ & $70,9 \%$ \\
\hline VO24 & LP & $\begin{array}{l}\text { Levar em consideração as questões pessoais dos } \\
\text { funcionários }\end{array}$ & $88,2 \%$ & $53,8 \%$ \\
\hline VO25 & $\mathrm{AG}$ & Estrutura de controle e comunicação interna & $86,2 \%$ & $70,0 \%$ \\
\hline VO26 & LP & Poder e liberdade de tomar decisão & $85,7 \%$ & $60,9 \%$ \\
\hline $\mathrm{VO} 27$ & $\mathrm{DE}$ & Protocolo para a realização das tarefas & $84,3 \%$ & $68,1 \%$ \\
\hline VO28 & $\mathrm{AG}$ & Plano de estratégia com relação a reparação de erros & $84,2 \%$ & $61,0 \%$ \\
\hline VO29 & $\mathrm{AG}$ & $\begin{array}{l}\text { Regras específicas de como os funcionários têm acesso } \\
\text { aos cargos }\end{array}$ & $84,2 \%$ & $55,4 \%$ \\
\hline VO30 & $\mathrm{AG}$ & Processo de tomada de decisão & $82,7 \%$ & $62,0 \%$ \\
\hline VO31 & $\mathrm{AG}$ & Foco no cliente & $81,9 \%$ & $60,8 \%$ \\
\hline VO32 & $\mathrm{AG}$ & $\begin{array}{l}\text { Política de reconhecimento e premiação para o } \\
\text { desempenho dos melhores funcionários }\end{array}$ & $81,7 \%$ & $52,0 \%$ \\
\hline VO33 & $\mathrm{AG}$ & Personalidade de um membro da equipe & $78,0 \%$ & $47,8 \%$ \\
\hline VO34 & LP & Adoção de um estilo padrão de gestão & $76,8 \%$ & $53,5 \%$ \\
\hline VO35 & $\mathrm{DE}$ & Participação na tomada de decisão & $76,7 \%$ & $49,3 \%$ \\
\hline VO36 & $\mathrm{DE}$ & Concordância & $74,4 \%$ & $57,0 \%$ \\
\hline VO37 & $\mathrm{AG}$ & Regras definidas de competitividade & $72,9 \%$ & $39,2 \%$ \\
\hline VO38 & $\mathrm{DE}$ & Estabilidade no emprego & $64,3 \%$ & $38,5 \%$ \\
\hline VO39 & LP & Autoridade e hierarquia & $61,7 \%$ & $38,2 \%$ \\
\hline VO40 & $\mathrm{AG}$ & Resultados e lucros & $23,3 \%$ & $14,2 \%$ \\
\hline
\end{tabular}

Nota: AG: Alta Gerência; LP: Líder de Projetos; DE: Desenvolvedores

A partir desse resultado, um survey foi elaborado com consultores e avaliadores de iniciativas de MPS com o objetivo de associar os VOs, identificados na primeira fase, 
com as RMs. Porém, como os participantes do survey não estão familiarizados com os VOs (que são de outra área de conhecimento - ciências sociais), foi decidido realizar uma pesquisa na literatura técnica para identificar FCS de forma a ser o elo de ligação entre os VOs e as RMs. Assim, o objetivo foi dividido em duas etapas: (i) associar os VOs com os FCS e (ii) relacionar os FCS com as RMs. Os detalhes do survey podem ser obtidos em [Passos 2014].

Para identificar os FCS, foram analisados 4 trabalhos científicos: [Montoni e Rocha 2010], [Niazi et al. 2005], [Dyba 2000] e [Niazi 2012]. Após a união de todos os FCS dos 4 trabalhos, que resultaram em 67 fatores, foi realizado um estudo para verificar as similaridades entre eles. Assim, foram identificados 31 FCS similares, resultando em uma lista com 36 FCS.

Após a conclusão dessas duas etapas, um framework conceitual foi desenvolvido objetivando ajudar as organizações de software na implementação/manutenção de iniciativas de MPS.

Tabela 2. Parte das RMs dos fatores humanos, sociais e organizacionais

\begin{tabular}{|c|c|}
\hline \multicolumn{2}{|r|}{ FATOR HUMANO } \\
\hline \multicolumn{2}{|r|}{ Equipes de Trabalho: } \\
\hline RM1 & $\begin{array}{l}\text { Definir uma equipe de garantia da qualidade, para apoiar a implementação da iniciativa de MPS. A } \\
\text { equipe deve ser composta por pessoas que (i) possuem senso de coordenação, (ii) sejam experientes } \\
\text { e qualificadas, (iii) sejam reconhecidas por ter um bom envolvimento com a alta direção e (iv) } \\
\text { consigam integrar os funcionários nos objetivos da implementação da estratégia. Além disso, que } \\
\text { inspire confiança, respeito e autoridade nos funcionários. É necessário, que tenham conhecimento } \\
\text { em software que possam contribuir para a melhoria do processo. }\end{array}$ \\
\hline RM2 & $\begin{array}{l}\text { Definir as competências, funções e responsabilidades dos membros das equipes para realizar os } \\
\text { papéis na implementação da iniciativa de MPS. }\end{array}$ \\
\hline \multicolumn{2}{|r|}{ Relacionamento: } \\
\hline RM12 & Ter um relacionamento caracterizado pelo respeito mútuo, pela educação, amizade, simpatia e ajuda. \\
\hline RM13 & Escolher pessoas que tenham qualidades como paciência e experiência para trabalharem juntas. \\
\hline \multicolumn{2}{|r|}{ Características Pessoais: } \\
\hline RM18 & $\begin{array}{l}\text { Definir os líderes responsáveis pela implementação da iniciativa de MPS. Estes devem lidar com o } \\
\text { desenvolvimento e definição do estilo de gestão, do meio de comunicação e da interação social } \\
\text { dentro da organização. Além disso, devem ser pessoas com alta credibilidade dentro da organização, } \\
\text { com alto poder de liderança, organizados, ter sua competência reconhecida, ser bons coordenadores e } \\
\text { estarem disponíveis. }\end{array}$ \\
\hline RM20 & Assumir compromissos e concordar com o que foi definido. \\
\hline \multicolumn{2}{|r|}{ FATOR SOCIAL } \\
\hline \multicolumn{2}{|r|}{ Clientes: } \\
\hline RM25 & $\begin{array}{l}\text { Responder constantemente às necessidades dos clientes e estabelecer um relacionamento de } \\
\text { confiança com eles. }\end{array}$ \\
\hline RM26 & Expor suas ideias e propostas ao cliente, ao invés de aceitar tudo que ele propõe. \\
\hline \multicolumn{2}{|r|}{$\begin{array}{c}\text { Participacão: } \\
\end{array}$} \\
\hline RM28 & $\begin{array}{l}\text { Realizar reuniões periódicas dando ênfase aos beneficios que a organização tem adquirido com a } \\
\text { implementação da iniciativa de MPS e caracterizar a situação da organização no futuro. }\end{array}$ \\
\hline RM30 & $\begin{array}{l}\text { Promover a satisfação e o envolvimento do funcionário através de um planejamento cuidadoso, de } \\
\text { um ambiente disciplinado, exigente e desafiante, com oportunidades de crescimento e atribuições } \\
\text { coerentes. Além disso, levar em consideração o bem-estar dos funcionários, tendo a preocupação } \\
\text { com a carga de trabalho pesada e más condições de trabalho. }\end{array}$ \\
\hline \multicolumn{2}{|r|}{ Apoio: } \\
\hline RM36 & $\begin{array}{l}\text { Mostrar claramente o apoio na implementação da iniciativa de MPS e não ser contraditória nas } \\
\text { decisões e ações tomadas. }\end{array}$ \\
\hline RM38 & Contar com apoio de dentro e fora da organização. \\
\hline \multicolumn{2}{|r|}{ Comprometimento: } \\
\hline RM41 & $\begin{array}{l}\text { Conquistar a confiança, a contribuição e o compromisso dos funcionários, além de promover o } \\
\text { diálogo. Isso pode ser feito através da participação dos mesmos nas decisões sobre MPS. Consultar } \\
\text { os funcionários quando decisões mais importantes forem tomadas. Argumentar que o sucesso da } \\
\text { iniciativa depende da participação de todos e envolvê-los na implementação da iniciativa de MPS. }\end{array}$ \\
\hline
\end{tabular}




\begin{tabular}{|c|c|}
\hline RM42 & $\begin{array}{l}\text { Ter a capacidade de se adaptar dinamicamente a uma rápida mudança, até mesmo no mais caótico } \\
\text { ambiente. Adotar táticas apropriadas para o gerenciamento das mudanças ocorridas com a iniciativa } \\
\text { de MPS. Estar preparados para as mudanças, particularmente nas áreas de planejamento, controle, } \\
\text { procedimentos (principalmente para as definições de processos formais) e cultura (adotar um } \\
\text { conjunto de estratégias para gerir as mudanças ocasionadas pela implementação de MPS). }\end{array}$ \\
\hline \multicolumn{2}{|r|}{ Ambiente: } \\
\hline RM49 & $\begin{array}{l}\text { Propiciar um ambiente simples, aconchegante, organizado, descontraído, relaxante, acolhedor, } \\
\text { arrumado, limpo e com uma decoração elegante. }\end{array}$ \\
\hline RM50 & Definir um espaço próprio para os funcionários, que deve ser caracterizado com itens pessoais. \\
\hline \multicolumn{2}{|r|}{ FATOR ORGANIZACIONAL } \\
\hline \multicolumn{2}{|r|}{ Políticas Organizacionais: } \\
\hline RM54 & Estabelecer um canal de comunicação eficaz e formal em toda a organização. \\
\hline RM55 & $\begin{array}{l}\text { Estabelecer políticas organizacionais e administrativas para controle e acompanhamento das } \\
\text { atividades de MPS. Gerenciar os prazos e os resultados estabelecidos para a iniciativa de MPS. Os } \\
\text { resultados obtidos (qualitativos e quantitativos) devem ser publicados de modo que todos os } \\
\text { funcionários tenham acesso. }\end{array}$ \\
\hline \multicolumn{2}{|r|}{ Metas/Objetivos: } \\
\hline RM67 & $\begin{array}{l}\text { Definir, coletivamente, as metas, os objetivos, as estratégias e os recursos necessários para } \\
\text { implementação da iniciativa de MPS, tanto a curto quanto á longo prazo, e alinhá-los com os } \\
\text { objetivos e as estratégias da organização, deixando-os explícitos a todos os envolvidos na } \\
\text { organização, assegurando que estes compreendam a razão da iniciativa de MPS. Definir um } \\
\text { calendário de atividades da iniciativa de MPS. }\end{array}$ \\
\hline RM68 & $\begin{array}{l}\text { Medir e comunicar o andamento da implementação da iniciativa de MPS. Realizar as medições e as } \\
\text { avaliações de desempenho de forma confiável e com métodos válidos. }\end{array}$ \\
\hline \multicolumn{2}{|r|}{ Cultura Organizacional: } \\
\hline RM71 & Alinhar a cultura organizacional com o gerenciamento de projetos de software. \\
\hline RM73 & $\begin{array}{l}\text { Utilizar os insights da cultura organizacional para melhor implementarem a iniciativa de MPS, isto } \\
\text { é, assegurar que as práticas de MPS estejam de acordo com a cultura organizacional. }\end{array}$ \\
\hline \multicolumn{2}{|r|}{ Investimentos/Recursos: } \\
\hline RM78 & Investir em tecnologias, cursos e ferramentas que suportam a execução de iniciativas de MPS. \\
\hline RM80 & $\begin{array}{l}\text { Dar recompensas e benefícios, como dias de folga, reconhecimento ou itens materiais, para os } \\
\text { funcionários mais envolvidos e que contribuíram para a iniciativa. }\end{array}$ \\
\hline \multicolumn{2}{|r|}{ Planos/Planejamentos: } \\
\hline RM87 & $\begin{array}{l}\text { Ter um plano estratégico para resolver problemas que possam ocorrer com a iniciativa de MPS. } \\
\text { Neste plano devem constar ações de intervenção, de assistência e garantia de melhores condições de } \\
\text { trabalho. Além disso, analisar fatores situacionais em termos dos riscos enfrentados por uma equipe } \\
\text { de MPS. }\end{array}$ \\
\hline RM88 & $\begin{array}{l}\text { Ter um plano de estabilidade no emprego (plano de carreira) para os funcionários através da } \\
\text { definição de regras e divulgar entre eles. }\end{array}$ \\
\hline
\end{tabular}

\section{Framework Conceitual de Apoio à Iniciativas de MPS a partir da CO}

O framework conceitual proposto neste trabalho é constituído de conceitos e relacionamentos fundamentados em um conjunto de proposições adquiridas nas duas fases de pesquisa descritas na Seção 3. O cenário de aplicação do framework são organizações de software que desejam ter conhecimento sobre o seu perfil cultural, visando contribuir com a alta direção e gerentes no sentindo de propiciar uma reflexão/análise dos VOs que estão inseridos nela. Deste modo, a organização pode estabelecer suas políticas e práticas organizacionais, ter conhecimento das atitudes e comportamento dos funcionários e entender o seu modo de funcionamento. O principal objetivo do framework é sugerir recomendações de melhoria a partir da análise dos VOs inseridos na organização, visando apoiar a implementação de iniciativas de MPS.

Na Figura 2 é apresentada uma visão geral do framework conceitual, onde primeiramente é realizada uma pesquisa com a organização de software visando identificar os VOs (1. VOs da Organização de Software). O resultado dessa pesquisa é processado juntamente com dois repositórios (2. Nível de Relevância dos VOs para Iniciativas de MPS e 3. Relacionamento dos VOs x RMs), que foram construídos conforme explicado 
na Seção 3. Uma análise (4. Análise dos Indicadores para Cálculo do Diagnóstico Organizacional) é realizada a partir desse processamento tendo como resultado um diagnóstico organizacional (5. Diagnóstico Organizacional da CO e RMs para Iniciativas de MPS) que sugere RMs para apoiar a implementação de iniciativas de MPS.

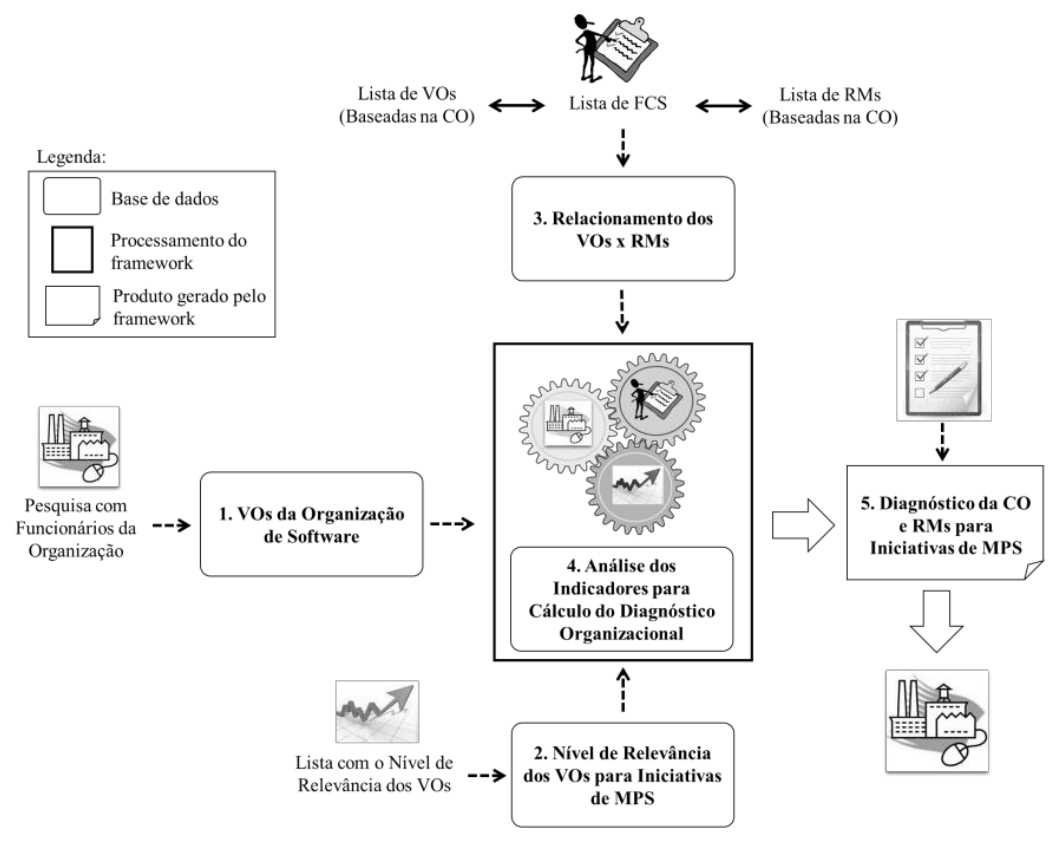

Figura 2. Visão geral do framework conceitual

A Figura 3 apresenta o detalhamento das etapas do framework, desde a pesquisa com os funcionários (Fase 1 da Figura 2) na organização de software até a geração do produto final do framework, que é o Diagnóstico Organizacional da CO e RMs para iniciativas de MPS (Fase 5 da Figura 2). Para representar esse framework, foi utilizado o meta-modelo SPEM (Software Process Engineering Metamodel), proposto pela Object Management Group [OMG 2008], que expressa a descrição de um processo de software. Todas as etapas do framework conceitual estão descritas a seguir.

Na Etapa 1 (Extrair os VOs por meio da opinião dos funcionários) é realizada uma pesquisa com as organizações de software, onde os funcionários respondem um questionário contendo 40 VOs com opções de respostas baseadas em uma escala de Likert de cinco pontos (Sem Opinião, Discorda Totalmente, Discorda, Concorda, Concorda Totalmente), indicando o grau de concordância quanto aos VOs que permeiam na organização de software em que atuam. Além disso, os funcionários respondem 6 questões objetivas referentes ao seu perfil profissional.

$\mathrm{Na}$ Etapa 2 (Descrever o perfil dos funcionários), é descrito um resumo contendo o perfil de todos os funcionários que responderam à pesquisa, conforme resultados obtidos no Etapa 1.

Na Etapa 3 (Calcular os índices positivo e negativo da organização) é calculado o índice positivo e negativo da organização conforme os resultados obtidos na Etapa 1. Esses índices são calculados da seguinte forma: será rotulado como resposta "POSITIVO" a soma do número de respostas das opções "Concorda" e "Concorda Totalmente". De forma similar, a soma do número de respostas com as opções "Discorda" e "Discorda Totalmente" serão rotuladas como "NEGATIVO". Como 
resultado, é exibida a porcentagem em relação às opções POSITIVO e NEGATIVO para cada VO, através de gráficos. Além disso, serão tabelados os VOs que apresentam uma situação mais crítica na organização (índice NEGATIVO maior que o índice POSITIVO). O objetivo desta análise é apresentar aos diretores e gerentes os VOs que são menos praticados/evidentes na organização segundo a opinião dos funcionários. $\mathrm{O}$ nível da organização (NO) para um VO corresponde ao índice POSITIVO, ou seja, é o percentual de respostas das opções "Concorda" e "Concorda Totalmente".

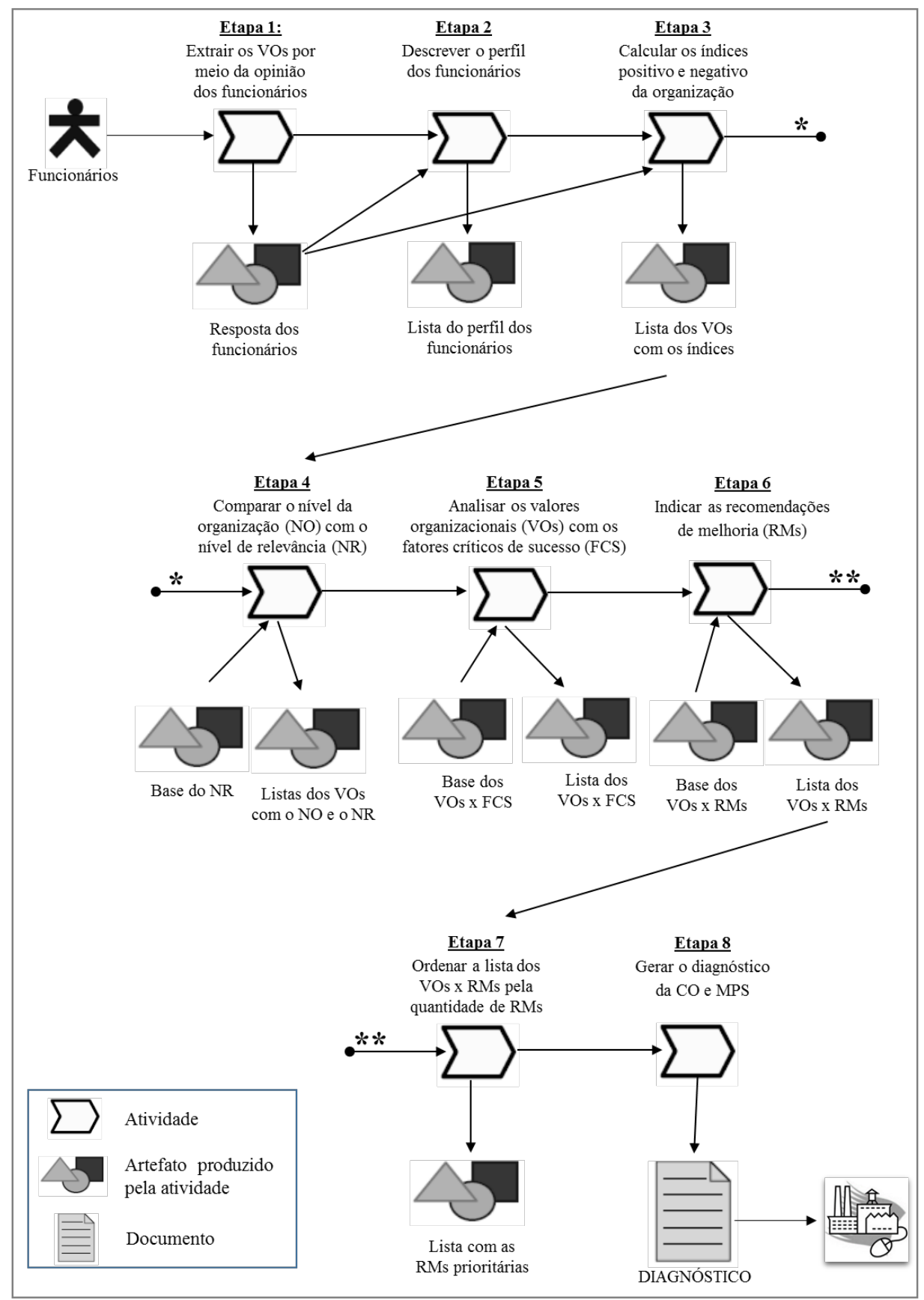

Figura 3: Sequência de passos para aplicação do framework conceitual 
Na Etapa 4 (Comparar o nível da organização (NO) com o nível de relevância (NR)) é feita uma análise do nível de relevância (NR) dos VOs para iniciativas de MPS (obtido conforme descrito na Seção 3) com o nível da organização (NO). O objetivo dessa análise é facilitar a visualização de quais VOs estão com o NO abaixo ou acima do NR e que precisam de recomendações para serem implantados na organização,

Na Etapa 5 (Analisar os VOs com os FCS) é utilizado um repositório (VOs x FCS), conforme discutido na Seção 3. Como resultado, são apresentadas 3 tabelas, por grupo de sucesso (Fator Humano, Fator Social e Fator Organizacional), com os VOs nos quais o NO está com avaliação abaixo do NR. Além disso, são mostrados os FCS que estão associados a esses VOs. Antes de serem apresentadas as 3 tabelas, a organização precisa definir o ponto de corte para os FCS de modo que atenda às suas necessidades. Esse ponto de corte é em função de um valor percentual (variando de $10 \%$ a $90 \%$ ), significando que quanto menor o valor desse percentual, mais FCS serão apresentados. O objetivo dessa análise é mostrar, por grupo de sucesso, os FCS que estão associados aos VOs nos quais o NO está abaixo do NR.

Na Etapa 6 (Indicar as RMS) são utilizados dois repositórios (VOs x FCS e FCS $\mathrm{x}$ RMs) que definem o repositório VO x RMs, conforme discutido na Seção 3. Esses dois repositórios são necessários, pois o ponto de corte está relacionado aos FCS (do primeiro repositório) e as RMs (do segundo repositório). Logo, assim como no passo anterior, quanto menor o valor percentual do ponto de corte, mais RMs serão sugeridas. Como resultado, é apresentada uma tabela com as RMs que estão relacionadas aos VOs nos quais o NO está com avaliação abaixo do NR. Vale ressaltar que as RMs selecionadas para fazerem parte dessa tabela deverão pertencer ao mesmo grupo de sucesso dos VOs identificados com o NO abaixo do NR.

Na Etapa 7 (Ordenar a lista dos VOs x RMs pela quantidade de RMs), a tabela gerada na etapa é mostrada de outra maneira, de forma a ser possível apresentar as RMs que podem ser prioritariamente implantadas na organização objetivando resolver, com um esforço mais gradativo (poucas RMs por vez), um maior número possível de VOs.

Na Etapa 8 (Gerar o diagnóstico organizacional da CO e MPS) é gerado o Diagnóstico Organizacional da CO e RMs para Iniciativas de MPS (DCO_MPS) que é um relatório contendo as etapas descritas acima visando relatar uma pesquisa realizada com funcionários de uma organização de software e sugerir RMs, através da análise dos VOs, que podem contribuir com a implementação de uma iniciativa de MPS. Um modelo real do DCO_MPS, realizado com uma organização de software, pode ser visto em [Passos 2014].

A próxima Seção apresenta os resultados das avaliações realizadas com o framework proposto.

\section{Avaliação do Framework Conceitual}

Além dos estudos conduzidos na primeira e segunda fases da pesquisa para compor o framework conceitual, outros dois estudos foram planejados e executados para: (i) avaliar o modelo para extrair os VOs e (ii) avaliar o diagnóstico organizacional da $\mathrm{CO}$ e RMs para iniciativas de MPS provido pelo framework.

\subsection{Avaliação do Modelo para Extrair os Valores Organizacionais}

Uma pesquisa de opinião foi planejada e executada com o objetivo de avaliar se o questionário para definir quais os VOs permeiam na organização de software, aplicado 
com os funcionários na Etapa 1, é claro o suficiente para ser aplicado em organizações de software e, assim, compor o framework proposto.

Participaram da pesquisa 4 organizações de software (OS1, OS2, OS3 e OS4), localizadas nas cidades de Manaus, Maceió e Rio de Janeiro, por meio de seus diretores, líderes/gerentes de projetos e membros da equipe técnica. Como instrumentação do estudo foi utilizado um formulário on-line composto de 10 questões, sendo 7 objetivas e 3 subjetivas. Além dessas questões, foi solicitado o perfil da organização.

No total, 70 funcionários participaram da pesquisa, sendo 9 da OS1, 12 da OS2, 31 da OS3 e 18 da OS4. Destes, 64,3\% dos funcionários já conheciam o assunto abordado, indicando que muitos já ouviram falar da CO e VOs. As instruções de como preencher o questionário foram satisfatórias para $90 \%$ dos funcionários. A forma como o questionário foi estruturado permitiu uma análise correta para a maioria $(75,7 \%)$ dos funcionários, e 84,3\% deles não tiveram nenhuma dificuldade para preenchê-lo.

As 40 afirmações sobre os VOs, como por exemplo: "A organização adota uma estratégia para gerenciamento dos riscos", "O líder/gerente de projeto realiza um acompanhamento das atividades planejadas" e "Os membros da equipe tem responsabilidades quanto a prazos e metas", foram claras e coerentes, facilitando o entendimento, para $84,3 \%$ dos funcionários. Essas afirmações, segundo $77,1 \%$ dos funcionários, podem dar uma ideia de como a organização funciona. Além disso, cerca de $72 \%$ dos funcionários acham viável utilizar esse modelo de questionário para avaliar os VOs de uma organização.

Portanto, o questionário aplicado foi bem aceito e com questões coerentes, de acordo com a opinião da maioria dos respondentes, podendo ser aplicado no contexto de análise dos VOs. Os detalhes da pesquisa podem ser obtidos em [Passos 2014].

\subsection{Avaliação do Diagnóstico Organizacional}

Para realizar a avaliação do DCO_MPS foram selecionados 10 avaliadores e/ou consultores experientes em atuar com iniciativas de MPS. A avaliação envolveu a realização de entrevistas semi-estruturadas guiadas por um questionário composto por 17 questões objetivas e 1 subjetiva, permitindo ao entrevistado expor suas opiniões e argumentos. Este questionário foi desenvolvido especificamente para explorar a forma de como o DCO_MPS foi estruturado.

As entrevistas foram realizadas nos meses de outubro e novembro de 2013, onde o pesquisador teve a oportunidade de conversar pessoalmente com todos os participantes e pôde discutir todos os detalhes do DCO_MPS. Dos 10 participantes selecionados, apenas um não pôde participar das entrevistas, totalizando assim a participação de 9 avaliadores e/ou consultores de MPS. A Tabela 3 apresenta a caracterização dos participantes. Detalhes da pesquisa podem ser obtidos em [Passos 2014].

Como resultado da pesquisa, para 90\% dos entrevistados o DCO_MPS está claro, coerente e reflete os VOs e pontos de carência da organização a serem evoluídos e $100 \%$ concordam que o DCO_MPS é pertinente para auxiliar a organização no entendimento dos VOs e no auxílio à implementação de iniciativas de MPS sob o aspecto da CO. Além disso, recomendariam o diagnóstico para organizações de software que desejam implementar/evoluir iniciativas de MPS.

Outro ponto positivo do diagnóstico, para $100 \%$ dos entrevistados, é apresentar os FCS e distribuir os VOs em categorias (Organização, Líder/Gerente de Projetos e Membros da Organização), pois facilita a organização no entendimento dos VOs. 
Tabela 3. Caracterização dos participantes da pesquisa

\begin{tabular}{|c|c|c|c|c|}
\hline ID & $\begin{array}{c}\text { Formação } \\
\text { Acadêmica }\end{array}$ & $\begin{array}{c}\text { Experiência } \\
\text { em MPS }\end{array}$ & $\begin{array}{c}\text { Programas/Normas que tem } \\
\text { Experiência }\end{array}$ & $\begin{array}{c}\text { Número de Organizações } \\
\text { que já Trabalhou }\end{array}$ \\
\hline 1 & Doutorado & Alta & MPS.BR, CMM/CMMI, Outros & 30 \\
\hline 2 & Doutorado & Excelente & MPS.BR, CMM/CMMI, ISO 12207 & 20 \\
\hline 3 & Mestrado & Alta & $\begin{array}{c}\text { MPS.BR, CMM/CMMI, ISO 15504, } \\
\text { ISO 12207, PMBOK, Outros }\end{array}$ & 15 \\
\hline 4 & Mestrado & Alta & $\begin{array}{c}\text { MPS.BR, CMM/CMMI, } \\
\text { ISO 15504, ISO 12207, Outros }\end{array}$ & - \\
\hline 5 & Mestrado & Alta & MPS.BR & 15 \\
\hline 6 & Doutorado & Excelente & $\begin{array}{c}\text { MPS.BR, CMM/CMMI, } \\
\text { ISO 15504, ISO 12207, PMBOK }\end{array}$ & 6 \\
\hline 7 & Mestrado & Alta & MPS.BR, CMM/CMMI & 8 \\
\hline 8 & Mestrado & Alta & $\begin{array}{c}\text { MPS.BR, CMM/CMMI, ISO 15504, } \\
\text { ISO 12207, PMBOK }\end{array}$ \\
\hline 9 & Doutorado & Alta & MPS.BR, CMM/CMMI & - \\
\hline
\end{tabular}

Sugestões de melhorias também foram providas pelos entrevistados. Como por exemplo, melhorar o texto sobre os níveis de importância e relevância dos VOs para que os gerentes das organizações não tenham dificuldades de entender o que está exposto no diagnóstico e mudar o gráfico de linha para outro tipo de gráfico, como o de coluna ou radar. Todas as sugestões e indicações de melhorias para o modelo do DCO_MPS foram discutidas e, após todas as considerações, as modificações foram realizadas, gerando uma nova versão do DCO_MPS.

Além da avaliação com os 9 consultores/avaliadores de iniciativas de MPS, foram realizadas 2 avaliações com as organizações de software OS1 e OS2. Após o envio do DCO_MPS para essas organizações com o diagnóstico personalizado para seus perfis culturais, foi enviado um e-mail solicitando à direção que preenchesse um questionário para avaliá-lo. A Tabela 4 mostra os resultados obtidos com essas duas organizações sobre a avaliação do DCO_MPS.

\subsection{Ameaças à Validade}

Algumas ameaças a validade relacionadas a ambas as avaliações (nas empresas e com avaliadores) podem ser classificadas em validades interna e externa:

Validade Interna: o primeiro estudo propõe utilizar membros de organizações de software com iniciativas de MPS em andamento e o segundo estudo foi conduzido com pesquisadores que são consultores e avaliadores de iniciativas de MPS. Assume-se que essa população é representativa no que concerne a indivíduos que atuam no contexto de software, que estejam envolvidos com uma iniciativa de MPS e podem opinar sobre o framework proposto e o conteúdo apresentado no diagnóstico organizacional.

Validade Externa: As organizações que participaram do estudo foram selecionadas por conveniência, porém representam perfis diferentes, entre organizações iniciantes em iniciativas de MPS e outras com mais experiência. Entre os pesquisadores consultados durante a avaliação, eles estão incluídos entre os principais pesquisadores nacionais no assunto (atuando no comité de grandes eventos, como SBQS e SBES). Assim, assume-se que eles são representativos. A quantidade de participantes de ambos os estudos foi significativa (70 profissionais das organizações e 10 pesquisadores). Contudo, seria interessante aumentar o número de organizações a serem consultadas, aumentando também a representatividade junto à população geral de organizações com interesse em iniciativas de MPS. 


\section{Considerações Finais}

Diversas empresas desenvolvedoras de software estão preocupadas em melhorar a qualidade de seus produtos e na entrega de acordo com o orçamento e o prazo estabelecido. Uma das possíveis soluções é a adoção de uma iniciativa de MPS. Porém, somente algumas organizações conseguem implementar com sucesso uma iniciativa de MPS, devido a aspectos organizacionais, dentre os quais a $\mathrm{CO}$, que pode interferir no sucesso da implementação de uma dessas iniciativas.

Tabela 4. Resultado obtido sobre a avaliação do DCO_MPS pelas organizações

\begin{tabular}{|l|c|l|}
\hline \multicolumn{1}{|c|}{ Questões do Questionário } & OS1 & \multicolumn{1}{|c|}{ OS2 } \\
\hline O texto do diagnóstico está claro e coerente? & Sim & Sim. Texto bem objetivo \\
\hline $\begin{array}{l}\text { O diagnóstico é pertinente para auxiliar a } \\
\text { organização no entendimento dos valores } \\
\text { organizacionais? }\end{array}$ & Sim & $\begin{array}{l}\text { Sim. Iremos definir ações para os } \\
\text { pontos negativos identificados no } \\
\text { diagnóstico }\end{array}$ \\
\hline $\begin{array}{l}\text { O diagnóstico, de modo geral, reflete os valores } \\
\text { organizacionais intrínsecos da organização? }\end{array}$ & Sim & $\begin{array}{l}\text { Sim. Resultado do diagnóstico } \\
\text { alinhado com o resultado da } \\
\text { Avaliação MPS, que ocorreu em } \\
\text { Setembro de 2013 }\end{array}$ \\
\hline O diagnóstico atendeu às suas expectativas? & Sim & Sim. Superou as expectativas \\
\hline $\begin{array}{l}\text { Você recomendaria a pesquisa para outras } \\
\text { organizações de software? }\end{array}$ & Sim & $\begin{array}{l}\text { Sim. Oportunidade de melhoria para } \\
\text { as organizações }\end{array}$ \\
\hline $\begin{array}{l}\text { Você tem alguma sugestão para melhorar o } \\
\text { diagnóstico? }\end{array}$ & - & $\begin{array}{l}\text { Melhorar a legenda dos gráficos, } \\
\text { indicando explicitamente o que } \\
\text { compõe o ponto positivo e negativo }\end{array}$ \\
\hline Comentários Adicionais & - & \begin{tabular}{l} 
Parabéns pela pesquisa! \\
\hline
\end{tabular} \\
\hline
\end{tabular}

Uma alternativa é verificar, em trabalhos publicados, recomendações de melhoria, ou relatos de experiências, que podem fornecer possíveis orientações relacionadas com a implementação dessas iniciativas. Nesse sentindo, foi realizado pesquisas que pudessem agregar a $\mathrm{CO}$ com RMs.

Diante disso, este trabalho apresentou o desenvolvimento de framework conceitual, construído a partir de estudos primários e secundários, visando a proposição de um diagnóstico organizacional que forneça recomendações de melhoria, baseadas na cultura organizacional, para apoiar positivamente a implementação/manutenção de iniciativas de melhoria de processo em organizações de software. Além disso, visa contribuir com a alta direção e gerentes no sentindo de propiciar uma reflexão/análise dos VOs que estão inseridos nela, auxiliar no estabelecimento das políticas e práticas organizacionais, propiciar o conhecimento das atitudes e comportamento dos funcionários e entender o modo de funcionamento da organização. Os resultados indicam que o framework é pertinente para auxiliar a organização no entendimento dos VOs e no auxílio a implementação de iniciativas de MPS.

As principais contribuições oferecidas por pesquisa à comunidade que trabalham com iniciativas de MPS são:

- Conjunto de 40 VOs extraídos de modelos para análise da CO com seus respectivos níveis de importância para uma organização de software e relevância para a implementação de iniciativas de MPS.

- Conjunto de 100 RMs propostas na literatura técnica que forma relacionadas com a CO através de FCS.

- Um framework conceitual, constituído de conceitos e relacionamentos 
fundamentados em um conjunto de proposições adquiridos, para análise da $\mathrm{CO}$ e sugestão de RMs para apoiar iniciativas de MPS.

Assim como qualquer pesquisa científica, algumas limitações foram observadas no decorrer deste trabalho:

- Diversos fatores podem influenciar na implementação de iniciativas de MPS, tais como fatores tecnológicos, sociais, organizacionais e humanos. No entanto, este trabalho foca apenas em aspectos organizacionais, como a $\mathrm{CO}$.

- Muitos são os modelos para análise da cultura organizacional. No entanto, este trabalho só identificou 36 modelos, selecionou 7 a partir de critérios estabelecidos, e deles extraiu 40 valores organizacionais.

- A maioria das RMs não foram avaliadas em organizações de software para verificar se promoveram ou não o sucesso na adoção/implementação da iniciativa de MPS, apesar de $45 \%$ delas apresentarem evidências de que foram avaliadas, sejam por pesquisa de opinião, estudos de casos ou revisões da literatura.

- Embora todas as etapas do framework conceitual estarem embasadas com estudos primários e secundários, é necessário realizar estudos de casos, com organizações de software e equipes de desenvolvimento, colocando em prática o framework conceitual a fim de verificar sua eficiência no apoio à implementação de iniciativas de MPS.

Apesar de não serem conclusivos, os dados obtidos nos estudos indicam que a abordagem é viável para o que se propôs realizar. Assim, como próximos passos estão previstos:

- Verificar, em estudos e trabalhos relacionados, como as RMs podem ser implantadas na organização de software.

- Desenvolver uma ferramenta computacional para dar apoio ao modelo proposto de forma a ficar acessível a qualquer organização de software que queira utilizálo. Esta ferramenta está desenvolvida, faltando sua avaliação em cenário real.

- Realizar avaliações, com organizações de software, do framework conceitual com o propósito de verificar sua aplicabilidade em relação ao perfil cultural e as RMs.

\section{Agradecimentos}

À Fundação de Amparo à Pesquisa do Estado do Amazonas (FAPEAM) pelo apoio financeiro através do processo $\mathrm{N}^{\circ} 01135 / 2011$ (PRONEX).

\section{Referências}

Barreto, A. e Rocha, A. (2012). Definição e Gerência de Objetivos de Software Alinhados ao Planejamento Estratégico. XI Simpósio Brasileiro de Qualidade de Software, p. 399-413.

Dyba, T. (2000). An Instrument for Measuring the Key Factors of Success in Software Process Improvement. Empirical Software Engineering, v. 5, n. 4, p. 357-390.

Dyba, T. (2002). Enabling Software Process Improvement: An Investigation of the Importance of Organizational Issues. Empirical Software Engineering, v.7, p.387-390.

Dyba, T. (2005). An Empirical Investigation of the Key Factors for Success in Software Process Improvement. IEEE Transactions on Software Engineering, v. 31, n. 5, p. 
410-424.

Montoni, M. e Rocha, A. (2010). Applying Grounded Theory to Understand Software Process Improvement Implementation. In 7th International Conference on the Quality of Information and Communications Technology, Portugal, p. 25-34.

Muller, S.; Kraemmergaard, P. e Mathiassen, L. (2009). Managing Cultural Variation in Software Process Improvement: A Comparison of Methods for Subculture Assessment. IEEE Transactions on Engineering Management, v. 56, n. 4, p. 584-599.

Muller, S.; Mathiassen, L. e Balshoj, H. (2010). Software Process Improvement as Organizational Change: A Metaphorical Analysis of the Literature Journal of Systems and Software, v. 83, n. 11, p. 2128-2146.

Nasir, M.; Ahmad, R. e Hassan, N. (2008). Resistance Factors in the Implementation of Software Process Improvement Project in Malaysia. Journal of Computer Science, v. 4, n. 3, p. 211-219.

Niazi, M. (2012). An Exploratory Study of Software Process Improvement Implementation Risks. Journal of Software Maintenance and Evolution: Research and Practice, v. 24, p. 877-894.

Niazi, M.; Wilson, D. e Zowghi, D. (2005). A Maturity Model for the Implementation of Software Process Improvement: An Empirical Study. The Journal of Systems and Software, v. 74, n. 2, p. 155-172.

OMG. (2008). Object Management Group. Software \& Systems Process Engineering Meta-Model Specification, versão 2.0. Disponível em: www.omg.org/spec/SPEM/2.0/PDF. Acesso em: 21 mai. 2014.

Passos, O.; Dias-Neto, A.; Barreto, R. (2012). Assessing the Relevance of Organizational Culture in Software Process Improvement Initiatives. In: Ibero-American Conference on Software Engineering (CIbSE), Buenos Aires, v. 15, p. 57-70.

Passos, O.; Dias-Neto, A. e Barreto, R. (2013). Uma Investigação sobre Recomendações de Melhoria Baseadas em Aspectos Culturais para Apoiar Iniciativas de Melhoria de Processo de Software. XII Simpósio Brasileiro de Qualidade de Software, p. 66-80.

Passos, O. (2014). Recomendações de Melhorias Baseadas na Cultura Organizacional para Iniciativas em Melhoria de Processo de Software. 2014. 346 f. Tese (Doutorado em Informática) - Universidade Federal do Amazonas, Manaus.

Robbins, S. (2005). Comportamento Organizacional. 11. ed. São Paulo: Pearson Prentice Hall.

Schein, E. (2009). Cultura Organizacional e Liderança. 3. ed. São Paulo: Atlas.

Seevers, B. (2000). Identifying and Clarifying Organizational Values. Journal of Agricultural Education, v. 41.

Shih, C-C. e Huang, S-J. (2010). Exploring the Relationship Between Organizational Culture and Software Process Improvement Deployment. Information \& Management, v. 47, p. 271-281.

Spínola, R.; Dias-Neto, A. e Travassos, G. (2008). Abordagem para Desenvolver Tecnologia de Software com Apoio de Estudos Secundários e Primários. In: Experimental Software Engineering Latin American Workshop, Salvador.

Webster, P. (2010). Why are Expectations of Grievance Resolution Systems not Me? A Multi-Level Eexploration of Three Case Studies in Australia. Custom Book Centre, University of Melbourne. 